\title{
Efficient design principle for interfacial charge separation in hydrogen-intercalated nonstoichiometric oxides
}

Zhenao Gu, ${ }^{\mathrm{a}, \mathrm{d},{ }^{\dagger}}$ Le Zhang, ${ }^{\mathrm{b},{ }^{\dagger}}$ Bo Wen, ${ }^{\mathrm{b}}$ Xiaoqiang An, ${ }^{\mathrm{a}, \mathrm{c}}$ Li-Min Liu, ${ }^{\mathrm{b},{ }^{*}}$ Xingzhong Cao, ${ }^{\mathrm{f}}$ Tao Chen, ${ }^{\mathrm{g}}$ Jing Zhang, ${ }^{,}$Junwang Tang, ${ }^{\mathrm{c}}$ Huijuan Liu, ${ }^{\mathrm{de}} \mathrm{e}$ Jiuhui Qu,${ }^{\mathrm{a}, \mathrm{d}}$

${ }^{a}$ Key Laboratory of Drinking Water Science and Technology, Research Center for Eco-Environmental Sciences, Chinese Academy of Sciences, Beijing 100085, China

${ }^{\mathrm{b}}$ Beijing Computational Science Research Center, Beijing 100193, China

E-mail:1imin.liu@csrc.ac.cn

${ }^{\mathrm{c}}$ Department of Chemical Engineering, University College London, Torrington Place, London, WC1E 7JE, UK

${ }^{\mathrm{d}}$ University of Chinese Academy of Sciences, Beijing 100049, China

${ }^{\mathrm{e}}$ State Key Laboratory of Environmental Aquatic Chemistry, Research Center for Eco-Environmental Sciences, Chinese Academy of Sciences, Beijing 100085, China

${ }^{\mathrm{f}}$ Multi-discipline Research Division, Institute of High Energy Physics, Chinese Academy of Science, Beijing 100049, China

${ }^{g}$ Beijing Synchrotron Radiation Facility (BSRF), High Energy Institute of Physics, Chinese Academy of Sciences, Beijing 100049, China

'Dr. Zhenao Gu and Dr. Le Zhang contributed equally to this work.

Abstract: Establishing effective strategies to boost the separation of interfacial charge carriers is necessary to address the limiting bottlenecks of photocatalysis. Herein, a design principle of hydrogen-intercalated nonstoichiometric oxides is exemplified by $\mathrm{WO}_{3}$ photoanodes for efficient charge separation. By modulating the spatial distribution of the oxygen-deficient structures and hydrogenated charge extraction layer, a remarkable photocurrent density of $2.94 \mathrm{~mA} \cdot \mathrm{cm}^{-2}$ at $1.23 \mathrm{~V}$ vs. RHE was achieved, leading to $\mathrm{xx} \%$ of the maximum theoretical photocurrent of $\mathrm{WO}_{3}$ photoanodes apart from a $100 \mathrm{mV}$ cathodic shift in the onset potential. Both experimental observations and theoretical calculations illustrate the synergetic contributions of the photoelectric field-assisted rearrangement of the oxygen vacancy defects and the hydrogen-intercalated charge extraction layer to the electron migration and charge separation, i.e., nearly $93 \%$ of charges separated spatially by the novel design strategy. Our work thus provides new pathway for atomic-level modulation of interfacial layers and sheds light on designing high-performance nonstoichiometric 
nanoarchitectures for a wide range of applications.

\section{Introduction}

One of the challenging issues in photocatalysis and photoelectrocatalysis is ensuring fast and efficient charge separation and transfer in photocatalysts. Despite tremendous efforts have devoted to modulating the behavior of charge carriers, the applications of photocatalysis are still limited by the moderate power conversion efficiencies of photocatalysts.[1] Conventional systems overwhelmingly focus on the principle of a space charge layer with idealized interfacial structures, but the impacts of complicated factors (such as the surface states, dipolar field and molecular adsorption) on the photocarrier dynamics at the solid/liquid interface remain largely unknown.[2,3] In this regard, a new design principle for interfacial charge extraction is urgently needed.

Since the first report of black titania, oxygen vacancy $\left(\mathrm{V}_{\mathrm{o}}\right)$ modulation has triggered an explosion of interest with respect to emergent conduction behavior and enhanced charge separation.[4,5] Although many efforts are being pursued in this direction, controversy persists regarding the real role of oxygen vacancy defects and, in most cases, the defects are considered as detrimental recombination centers.[6,7] Recently, the crystalline/amorphous bilayer that results from the lattice distortion and atomic arrangement in oxygen-deficient semiconductors was validated, but a full atomistic picture of the oxygen vacancy layer is still missing.[8] To achieve this level of understanding, the impact of fundamental processes, such as the formation, drift and aggregation of vacancy defects, on the radical formation and interfacial charge separation must be determined.

Hydrogen is ubiquitous in material growth and reaction environments but is difficult to detect experimentally.[9-11] Most prevailing concerns about hydrogen-intercalated materials are focused on their electrochromic performance, and the impact of hydrogen on the interfacial separation of photoinduced charge carriers has been overlooked. The intercalation-induced surface reconstruction is accompanied by remarkable metallization and should strongly affect the electronic structure of semiconductors. $[12,13]$ The presence of hydrogen can also influence the adsorption 
and dissociation of water molecules, which is an essential prerequisite for surface hydroxylation. Thus, modulating hydrogenated structures has become a burgeoning strategy for collecting charge carriers.[14,15] Inspired by recent advances, we hypothesize that hydrogen-intercalated nonstoichiometric semiconductors can be created, which is an unexplored but important realm. The synergetic effect between the hydrogen-intercalated layer and the oxygen vacancy structures is expected to contribute to high-efficiency charge separation and unprecedented photoactivities.

In this work, our aim was to explicitly establish a design strategy for layer-dependent homo-interfaces for the efficient separation of charge carriers. Using $\mathrm{WO}_{3}$ as an example, a field-assisted methodology was used to control the spatial distribution of the oxygen stoichiometry and the hydrogenation layer, and the methodology consisted of spontaneous drift, depletion, depth rearrangement of the oxygen vacancies and manipulation of hydrogen intercalation. Due to the synergetic effect between the nonstoichiometric layer and the hydrogenated charge extraction layer, aremarkablephotocurrent density of $2.94 \mathrm{~mA} \cdot \mathrm{cm}^{-2}$ at $1.23 \mathrm{~V}$ vs. RHE was achieved with a marked $100 \mathrm{mV}$ cathodic shift in the onset potential. Both the experimental observations and theoretical calculation unequivocally indicated the formation of layer-dependent reactive species with specific reaction selectivity. The design principle of modulating oxygen (as vacancy defects) and hydrogen (as intercalated atoms) at the atomic level opens up new opportunities for photocatalysts. It is applicable to several semiconductors, and is important for the design of high-performance nanoarchitectures for a wide range of applications.

\section{Results and discussion}

Both defect control and hydrogen incorporation can be realized under an applied electric field, and exploring the possibility of field-assisted construction of multilayered interfaces for efficient charge separation is important.[16] As schematically illustrated in Figure 1a, well-aligned $\mathrm{WO}_{3}$ nanoflakes were used as a typical model to test this hypothesis. Four key procedures were involved: (i) the assembly of $\mathrm{WO}_{3}$ nanoflake arrays (Figure 1b); (ii) generation of a nonstoichiometric layer with oxygen vacancies under a hydrogen atmosphere (Video S1); (iii) 
photoelectric field-assisted rearrangement of the oxygen vacancies (Video S2); and (iv) electric field-induced intercalation of hydrogen to form the charge extraction layer (Video S3). Herein, the corresponding samples are denoted $\mathrm{WO}_{3}, \mathrm{~V}_{0}-\mathrm{WO}_{3}$, $\mathrm{R}-\mathrm{V}_{\mathrm{o}}-\mathrm{WO}_{3}$ and $\mathrm{H}-\mathrm{R}-\mathrm{V}_{\mathrm{o}}-\mathrm{WO}_{3}$, respectively.

The morphology observations (Figure S1andS2) confirm the in situ growth of 2 $\mu \mathrm{m}$ thick $\mathrm{WO}_{3}$ nanoflakes on tungsten plates with a preferential growth direction of [200]. The scanning transmission electron microscopy (STEM) images in Figure $1 \mathrm{c}-1 \mathrm{f}$ demonstrate the significant influences of the layer engineering processes on the surface structure of $\mathrm{WO}_{3}$. Compared with the pristine sample, the abstraction of lattice oxygen atoms in a hydrogen atmosphere results in the spontaneous formation of dispersed pits (Figure S3) and an ultrathin amorphous layer (as marked in Figure 1d). $[17,18]$ The reduced contrast in the centers of the unit cell indicate the formation of numerous oxygen vacancies in $\mathrm{V}_{\mathrm{o}}-\mathrm{WO}_{3}$. In contrast, the utilization of the photoelectric field facilitates the spatial rearrangement of the oxygen vacancies, as evidenced by the partially diminished pits and restored crystal lattices in $\mathrm{R}-\mathrm{V}_{\mathrm{o}}-\mathrm{WO}_{3}$ (Figure 1e and Figure S4). The slight lattice distortion around the retained pits proves the oxygen vacancies aggregate in the subsurface region (Figure 1e). When the electric field-assisted methodology was used to intercalate hydrogen into $\mathrm{WO}_{3}$, a thin, disordered layer adjacent to the distorted lattices can be observed in $\mathrm{H}-\mathrm{R}-\mathrm{V}_{\mathrm{o}}-\mathrm{WO}_{3}$ (Figure 1f). This indicates the construction of a charge extraction layer on top of the nonstoichiometric structures. Therefore, $\mathrm{WO}_{3}$ nanoflakes with distinguishable surface structures were obtained during the different layer modulation strategy processes, but their phase structure remained the same. (Figure S5). 


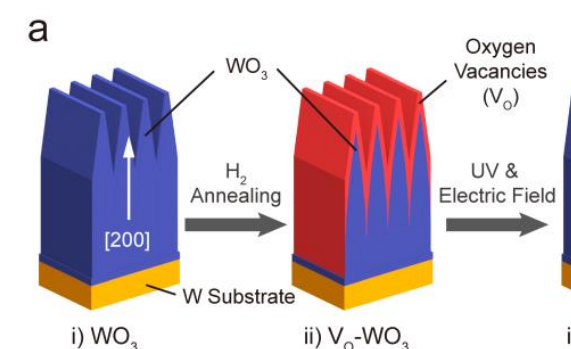
i) $\mathrm{WO}_{3}$

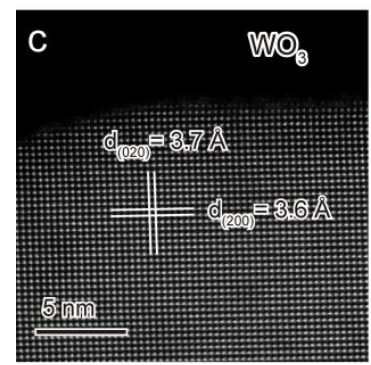

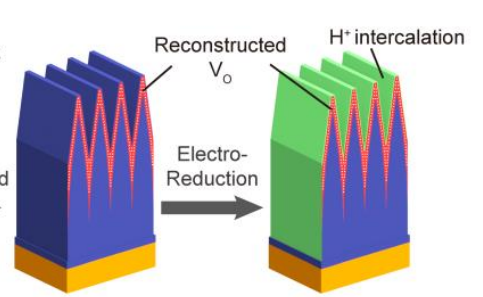

iv) $\mathrm{H}-\mathrm{R}-\mathrm{V}_{\mathrm{O}}-\mathrm{WO}_{3}$ b
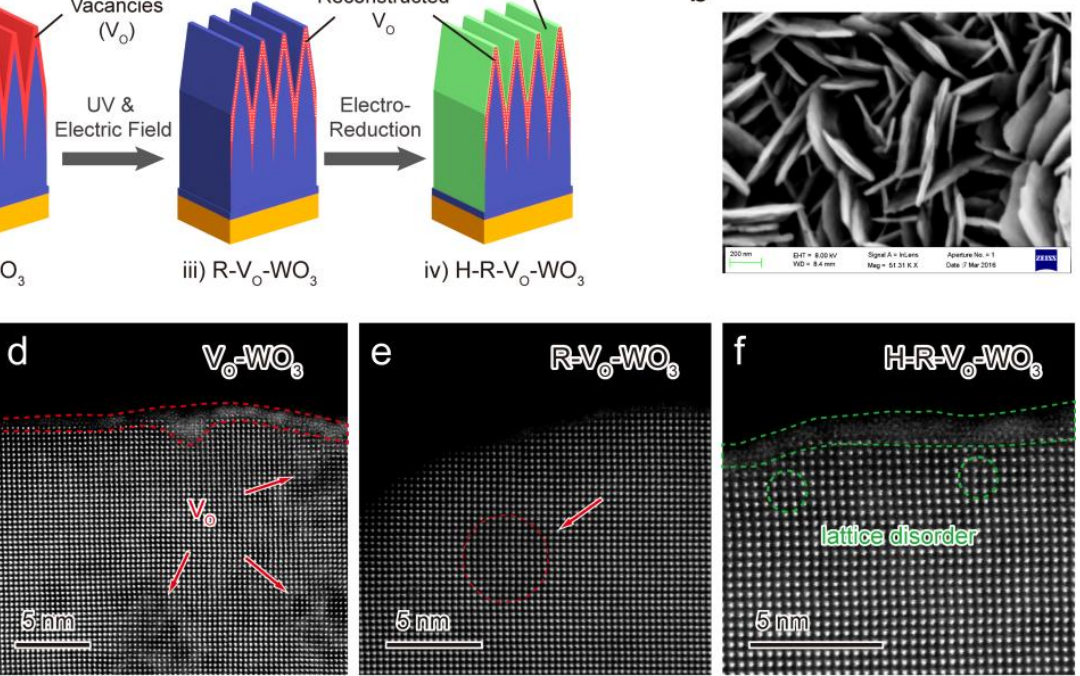

Figure 1 (a) Schematic illustration of the construction of hydrogen-intercalated, nonstoichiometric $\mathrm{WO}_{3}$ (Vo-WO3: surface oxygen vacancy rich WO3, R-Vo-WO3:xxx, H-R-Vo-WO3:xxx); (b) Top-view scanning electron microscopy (SEM) image of the $\mathrm{H}-\mathrm{R}-\mathrm{V}_{\mathrm{o}}-\mathrm{WO}_{3}$ nanoflake arrays; (c-f) STEM images of the corresponding samples (i-iv) in figure (a); the dash lines and arrows show the existence of $\mathrm{V}_{\mathrm{o}}$-induced structures. The red (e) and green (f) circled regions denote the slight lattice distortion caused by oxygen vacancies.

Photoelectrochemical (PEC) water oxidation experiments were performed to evaluate the impact of the surface modulation on the performance of the photoanodes. According to the photocurrent-density curves of the different samples, pristine $\mathrm{WO}_{3}$ had only a moderate photoactivity, with a platform photocurrent of $1.3 \mathrm{~mA} \cdot \mathrm{cm}^{-2}$ (Figure 2a). The generation of oxygen vacancies can contribute to the maximum photocurrent but deteriorate the onset potential by a positive shift of $135 \mathrm{mV}$. Encouragingly, a significantly improved PEC performance was achieved for $\mathrm{R}-\mathrm{V}_{\mathrm{o}}-\mathrm{WO}_{3}$, which indicated that the rearrangement of the oxygen vacancies suppressed the recombination of the charge carriers and affected the surface defect sites in $\mathrm{WO}_{3}$. As expected, $\mathrm{H}-\mathrm{R}-\mathrm{V}_{\mathrm{o}}-\mathrm{WO}_{3}$, which had a reconstructed nonstoichiometric layer and a hydrogen-intercalated charge extraction layer exhibited the highest photocurrent density of $3.52 \mathrm{~mA} \cdot \mathrm{cm}^{-2}$ at $1.8 \mathrm{~V}$ vs. RHE. This density is one of the highest values among those reported for $\mathrm{WO}_{3}$ photoanodes (Table S1). 
Compared to pristine $\mathrm{WO}_{3}$, the extremely high incident photocurrent efficiency (IPCE) at $1.23 \mathrm{~V}$ vs. RHE (Figure $2 \mathrm{~b}$ ) and the cathodically shifted onset potential of $100 \mathrm{mV}$ clearly confirmed the effectiveness of the multilayer modulation strategy.

To further elucidate this principle, the efficiency of the interfacial charge transfer was investigated. If a hole transfer efficiency of $100 \%$ in the presence of a $\mathrm{H}_{2} \mathrm{O}_{2}$ scavenger is assumed, the change in the photocurrent before and after the addition of $\mathrm{H}_{2} \mathrm{O}_{2}$ represents the contribution of the different layer structures to the water oxidation kinetics. The scavenging experiments clearly showed the remarkably improved photocurrent of $\mathrm{V}_{\mathrm{o}}-\mathrm{WO}_{3}$ at $1.23 \mathrm{~V}$ vs. RHE, whereas a negligible change was observed for $\mathrm{H}-\mathrm{R}-\mathrm{V}_{\mathrm{o}}-\mathrm{WO}_{3}$ (a hole transfer efficiency of $93 \%$ at $1.23 \mathrm{~V}$ vs. RHE in Figure 2c). These results demonstrated the near-complete elimination of the hole extraction limitation via hydrogen intercalation with a dramatically decreased onset potential. Due to the fast hole transport, the formation of the hydrogen-intercalated layer is also beneficial for the stability of the reconstructed oxygen-deficient $\mathrm{WO}_{3}$. Compared to the sharp decline in the photocurrent of pristine $\mathrm{WO}_{3}, \mathrm{H}-\mathrm{R}-\mathrm{V}_{\mathrm{o}}-\mathrm{WO}_{3}$ showed a steady photocurrent density even after $8 \mathrm{~h}$ of charge extraction (Figure $2 \mathrm{~d}$ ). Importantly, the slight decrease in the photocurrent caused by the release of hydrogen can be reversibly restored via facile and intermittent polarization at negative potentials, which suggests a regenerative layer structure for high-efficiency reactions. 
a

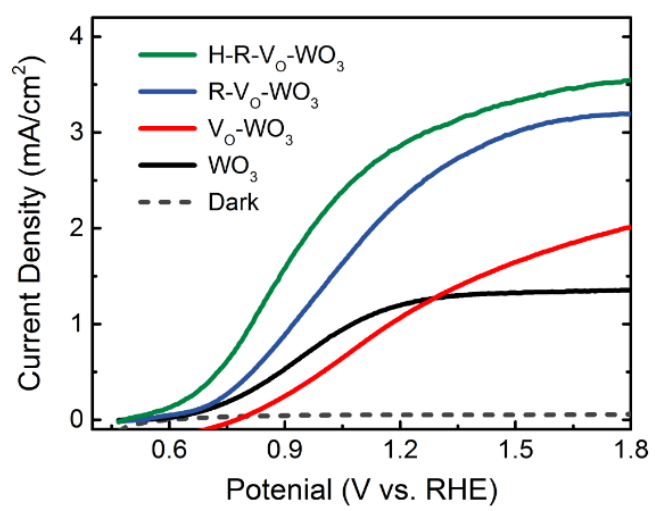

C

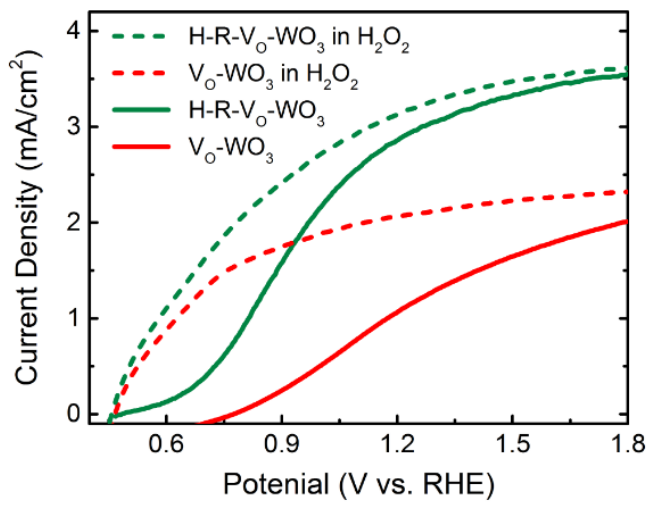

b

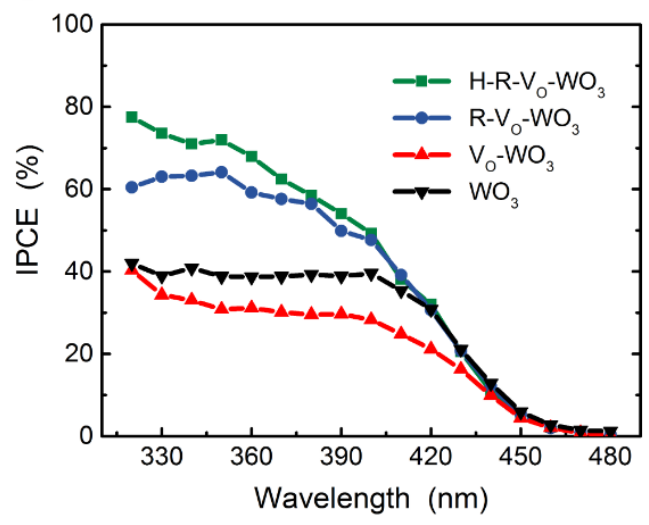

d

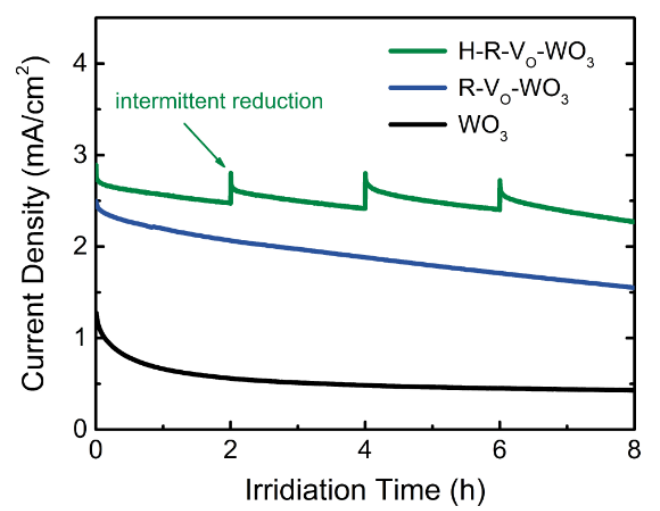

Figure 2 (a) $J-V$ curves of the $\mathrm{WO}_{3}$ photoanodes with different layer structures under AM 1.5G illumination, and $0.2 \mathrm{M} \mathrm{Na}_{2} \mathrm{SO}_{4}$ (ca. $\mathrm{pH} 7.0$ ) electrolyte; (b) The calculated photoconversion efficiencies of the $\mathrm{WO}_{3}$ photoanodes with and without interfacial layer modulation; (c)Photocurrent densities of $\mathrm{V}_{0}-\mathrm{WO}_{3}$ and $\mathrm{H}-\mathrm{R}-\mathrm{V}_{\mathrm{o}}-\mathrm{WO}_{3}$ with and without hole scavengers; (d) Time stability of the $\mathrm{H}-\mathrm{R}-\mathrm{V}_{\mathrm{o}}-\mathrm{WO}_{3}$ photoanode at $1.23 \mathrm{~V}$ vs. RHE.

To understand the impressive enhancement in the PEC behavior, X-ray photoelectron spectra (XPS) were used to study the structure of the nonstoichiometric layer. Valence band XPS confirmed the appearance of electron states above the valence band maximum (VBM) after treatment under a hydrogen atmosphere (Figure S6a). This result agrees well with the enhanced visible light absorption of $\mathrm{V}_{\mathrm{o}}-\mathrm{WO}_{3}$ in Figure S6b. Compared to pristine $\mathrm{WO}_{3}$, the prominent $\mathrm{W} 4 \mathrm{f}_{7 / 2}$ and $\mathrm{W} 4 \mathrm{f}_{5 / 2}$ peaks of $\mathrm{V}_{\mathrm{o}}-\mathrm{WO}_{3}$ shifted to lower energies, which suggested the formation of $\mathrm{W}^{5+}$ due to the oxygen vacancies (Figure S7a). However, the reverse shift of these peaks was observed after the field-induced rearrangement, which is consistent with the 
elimination of the surface oxygen vacancies in $\mathrm{R}-\mathrm{V}_{\mathrm{o}^{-}}-\mathrm{WO}_{3}$.[19] To gain fundamental insight into this redistribution process, the depth profiles of the oxygen vacancies were investigated via cathodoluminescence (CL). Because the excitation depth is strongly dependent on the incident energy of the electron beam, the relative concentration of the oxygen vacancies can be estimated using the ratio of the $\mathrm{V}_{\mathrm{o}}$-induced emission $(520 \mathrm{~nm})$ and the intrinsic luminescence $(420 \mathrm{~nm})$ (Figure 3a).[20] For a low energy excitation, the much stronger defect emission from $\mathrm{V}_{0}-\mathrm{WO}_{3}$ than that of pristine $\mathrm{WO}_{3}$ indicated the existence of sufficient oxygen vacancies in the surface region. However, a noticeabledecrease in the oxygen vacancies on the surface and in bulk with accumulated defects in the subsurface region was observed for $\mathrm{R}-\mathrm{V}_{\mathrm{o}}-\mathrm{WO}_{3}$ (Figure S8). Since bulk defects acting as recombination centers and surface defects acting as surface states are the two dominant limiting factors for nonstoichiometric photocatalysts, the reconstruction of the depth level of $V_{o}$ should contribute to charge separation in defective semiconductors.[21]

To establish a full picture of the electronic structure, nonstoichiometric $\mathrm{WO}_{3}$ before and after the defect rearrangement was further studied using grazing incidence X-ray absorption fine structure spectroscopy (GI-XAFS). A higher angle corresponds to a larger depth, and the distribution of the oxygen vacancies can be estimated by tuning the grazing incidence angle. At an incident angle greater than $20^{\circ}$, the white line peak of $\mathrm{R}-\mathrm{V}_{\mathrm{o}}-\mathrm{WO}_{3}$ was very similar to that of $\mathrm{V}_{\mathrm{o}}-\mathrm{WO}_{3}$ (Figure $3 \mathrm{~b}$ ), which is a clear sign of the bulk structure because the white line peak reflects the local crystal symmetry. With a decrease in the incidence angle, a notable intersection of the white line peaks appeared at $1.0^{\circ}$ for those two samples, which indicated that $\mathrm{R}-\mathrm{V}_{\mathrm{o}}-\mathrm{WO}_{3}$ has a relatively lower symmetry in the space charge region and a higher symmetry in the bulk compared to that of $\mathrm{V}_{\mathrm{o}}-\mathrm{WO}_{3}$. Combined with the absorption-edge energy results (Figure S9), we determined the sandwich-type distribution of the unsaturated, coordinated W caused by the oxygen vacancy rearrangement, as conceived in Figure $1 \mathrm{a}$.

In addition to the depth-dependent distribution, the field-induced rearrangement also has a significant impact on the chemical environment of the oxygen vacancies. 
Compared to the electron spin resonance (ESR) spectrum of $\mathrm{WO}_{3}$, the strong peak corresponding to $\mathrm{V}_{\mathrm{o}}$ at $\mathrm{g}=2.002$ in $\mathrm{V}_{\mathrm{o}}-\mathrm{WO}_{3}$ and the weakened signal in $\mathrm{R}-\mathrm{V}_{\mathrm{o}}-\mathrm{WO}_{3}$ (Figure 3c) prove the generation of and decrease in $\mathrm{V}_{\mathrm{o}}$ during step ii and step iii, respectively. Furthermore, the distinct, broad signal at $\mathrm{g}=2.07$ in $\mathrm{R}-\mathrm{V}_{\mathrm{o}}-\mathrm{WO}_{3}$ clearly demonstrates the formation of a new type of defect.[22] The changed chemical environment of the vacancy defects was further validated by the Doppler broadening spectra of the positron annihilation, which is also sensitive to the depth based on the positron incident energy.[23] Almost no difference was observed between the $\mathrm{S}$ parameters of $\mathrm{WO}_{3}$ and $\mathrm{V}_{0}-\mathrm{WO}_{3}$ (Figure 3d), which demonstrated the negligible influence of the increasing concentration on the status of the oxygen vacancies. However, $\mathrm{R}-\mathrm{V}_{\mathrm{o}}-\mathrm{WO}_{3}$ exhibited a much larger $\mathrm{S}$ parameter, especially in the relatively deep region, which indicated the formation of large vacancy clusters in the subsurface.[23] The positron annihilation lifetime spectra (PALS)further confirmed the existence of vacancy clusters in $\mathrm{R}-\mathrm{V}_{\mathrm{o}}-\mathrm{WO}_{3}$ (Figure $\mathbf{S 1 0}$ and Table S2). The evolution of the defect state could have an important effect on the separation behavior of the charge carriers. 
a

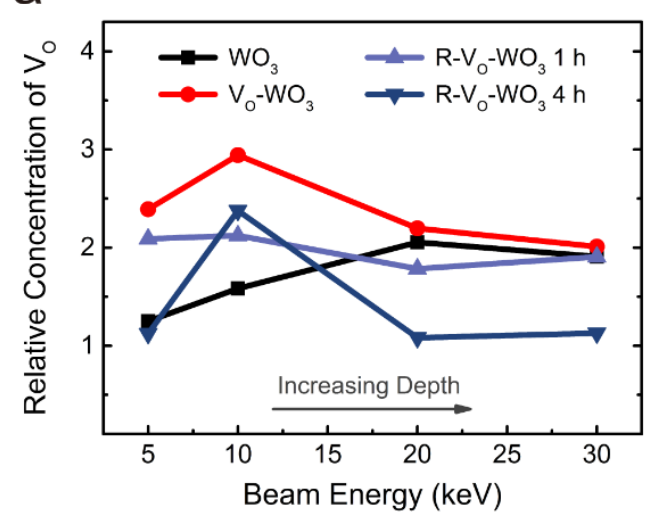

C

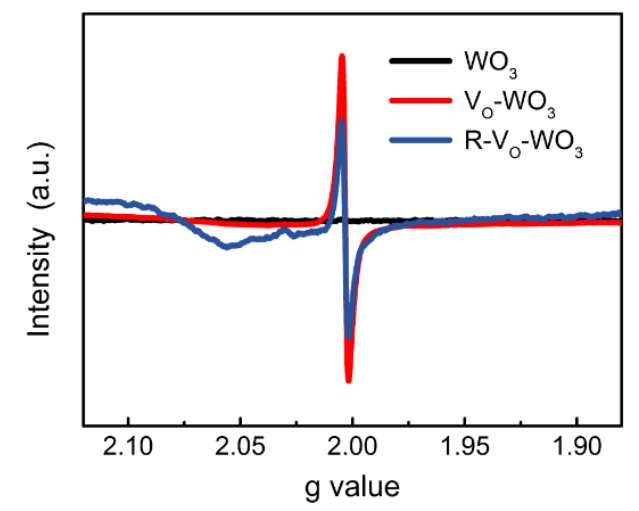

b

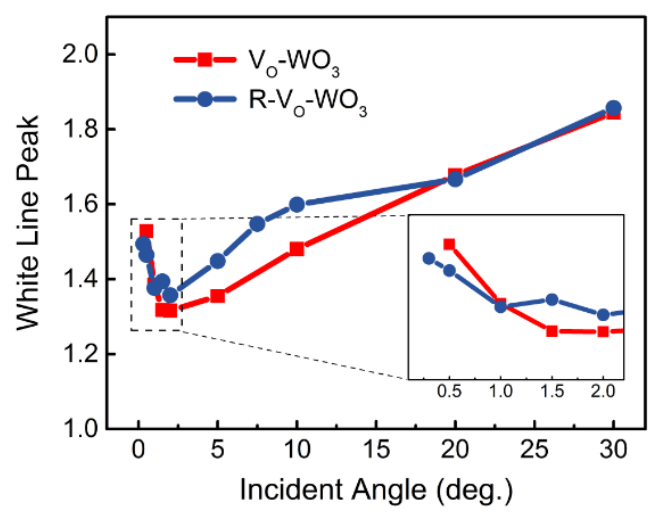

d

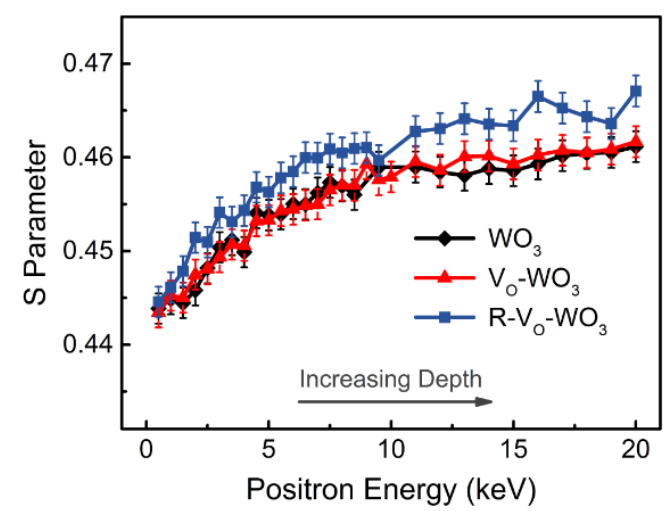

Figure 3 (a) Ratio of the $\mathrm{CL}$ peak area of the $\mathrm{WO}_{3}$ photoanodes before and after the structural rearrangement under different incident energies; (b) White line peak derived from the GI-XAFS of $\mathrm{V}_{\mathrm{o}}-\mathrm{WO}_{3}$ and $\mathrm{R}-\mathrm{V}_{\mathrm{o}}-\mathrm{WO}_{3}$; the inset shows the peak value at an incident angle of less than $5^{\circ}$; (c) ESR spectra of $\mathrm{WO}_{3}, \mathrm{~V}_{\mathrm{o}}-\mathrm{WO}_{3}$ and $\mathrm{R}-\mathrm{V}_{\mathrm{o}}-\mathrm{WO}_{3}$; (d) $\mathrm{S}$ parameter for different samples as a function of the positron energy.

The first principle calculations were performed to validate the migration property of $\mathrm{V}_{\mathrm{o}}$ (Figure 4a). According to the formation energies of $\mathrm{V}_{\mathrm{x}}(3.81 \mathrm{eV}), \mathrm{V}_{\mathrm{y}}(3.68 \mathrm{eV})$ and $\mathrm{V}_{\mathrm{Z}}(3.48 \mathrm{eV})$ on the surface, $\mathrm{V}_{\mathrm{Z}}$ is the preferential site for the formation of a single $V_{0}$. A similar trend was also observed for the formation of $V_{o}$ in the subsurface with a relatively higher formation energy (Figure S11). The small energy barrier of $0.98 \mathrm{eV}$ indicated that the as-formed $\mathrm{V}_{\mathrm{o}}$ is quite mobile and has a tendency to migrate from the subsurface to the surface, i.e., the depth-dependent reconstruction of $V_{o}$ defects in the experimental observations (Figure $4 \mathrm{~b}$ and Figure S12).

Based on the experimental characterizations and theoretical calculation, we mapped out the dynamic process of $\mathrm{V}_{\mathrm{o}}$, including the formation, field-induced diffusion and 
depth-dependent rearrangement (Figure 4c). The interruption of crystalline periodicity can lead to the formation of abundant $V_{o}$ defects. From a thermodynamic viewpoint, the drift of $V_{o}$ from deep in the bulk toward the surface is favorable.[24] Our research indicates that an electrical field, which is an emerging method for defect modulation, can provide a driving force to alleviate energy loss as an intrinsic potential barrier.[25,26] Therefore, the synergetic effect between the photo and electric fields is a prerequisite for overcoming the Fermi level pinning limitation in the inversion layer (Figure S13 and S14). With the rearranged oxygen vacancies in the depth level, the photoelectric field-assisted strategy can contribute to the significantly improved electron separation in $\mathrm{R}-\mathrm{O}_{\mathrm{v}}-\mathrm{WO}_{3}$, which was evidenced by the abruptly decreasing slope in the Mott-Schottky plot (Figure S13c).

a

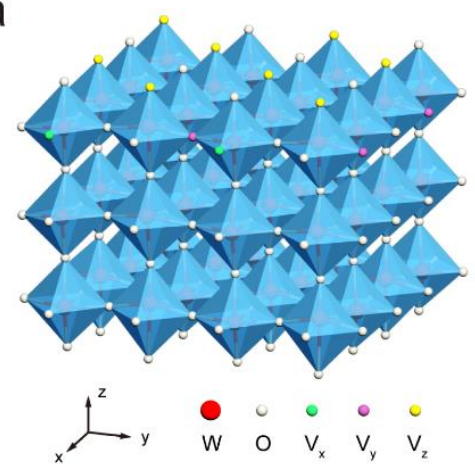

C

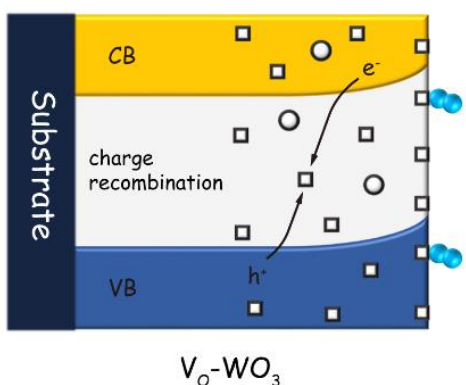

b
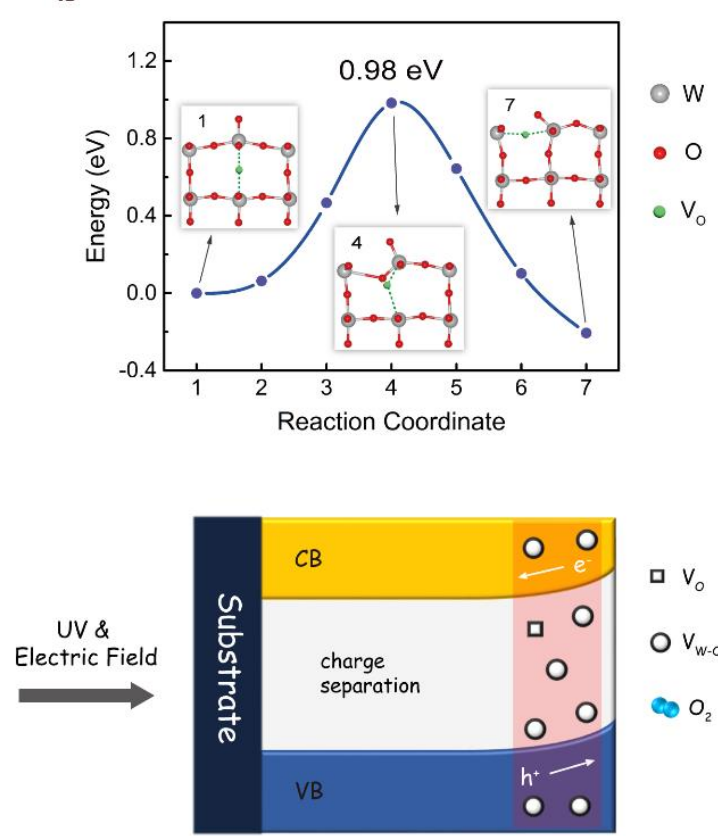

$\mathrm{R}-\mathrm{V}_{\mathrm{O}}-\mathrm{WO}_{3}$

Figure 4 (a) Top view of the $\mathrm{WO}_{3}(001)$ surface with oxygen vacancies formed at different sites; (b) The free energy variation of the whole system during the migration of one oxygen vacancy from the subsurface to the surface; (c) Schematic illustration of the field-induced oxygen vacancy rearrangement.

After determining the structure evolution of the oxygen vacancies, the formation of the hydrogen-intercalated layer on nonstoichiometric $\mathrm{WO}_{3}$ was then deliberated. Time of flight secondary ion mass spectrometry (ToF-SIMS) was used to determine the 
depth profiles of oxygen and hydrogen in the different samples. Compared to the pristine $\mathrm{WO}_{3}$ and $\mathrm{V}_{\mathrm{o}}-\mathrm{WO}_{3}, \mathrm{R}-\mathrm{V}_{\mathrm{o}}-\mathrm{WO}_{3}$ had more oxygen on the surface and less internal oxygen. This result supported the depth migration of the oxygen atoms to form a sandwich-type distribution of unsaturated, coordinated $\mathrm{W}$ after the rearrangement (Figure S15). However, the subsequent electrochemical reduction resulted in a significantly increased hydrogen signal in both the surface and inside $\mathrm{H}-\mathrm{R}-\mathrm{V}_{\mathrm{o}}-\mathrm{WO}_{3}$, which clearly demonstrates the easy intercalation of hydrogen into $\mathrm{WO}_{3}$ (Figure 5a). The $0.2 \mathrm{eV}$ shift of the W 4f XPS spectra to a lower binding energy (Figure S16) and the appearance of a hydroxyl peak at $531.7 \mathrm{eV}$ in the $\mathrm{O} 1 \mathrm{~s}$ spectra (Figure 5b) confirmed the hydrogen-induced amorphization and the corresponding structural change in the coordination polyhedron. This result agrees well with the Raman and Fourier transform infrared spectroscopy (FT-IR) results in Figure S17.

Next, we studied the interfacial charge separation behavior in the hydrogen-intercalated nonstoichiometric $\mathrm{WO}_{3}$. The formation of the hydrogen-intercalated charge extraction layer contributed to a decreased onset potential, enhanced photoactivity, and a more than one order of magnitude higher carrier density than that of pristine $\mathrm{WO}_{3}$ (Figure S18). Based on the significantly lowered charge transfer resistance and increased capacitance in the equivalent circuit of the Nyquist plots (Figure S19), the fundamental role of the hydrogen-intercalated layer as the surface state can be hypothesized (Figure S20 and S21).[27] As illustrated in Figure S22, the layer provides a bypass for holes to reach the semiconductor/liquid interface and facilitates the reaction kinetics. Thus, the superior PEC performance of $\mathrm{H}-\mathrm{R}-\mathrm{V}_{\mathrm{o}}-\mathrm{WO}_{3}$ can be attributed to the synergetic effect between the oxygen vacancy layer and the hydrogen-intercalated charge extraction layer. In other words, the oxygen vacancy layer contributes to fast electron migration across the film by eliminating the carrier transport limitations, and the holes can be efficiently extracted to the hydrogen-intercalated layer, i.e., spatial separation of the charge carriers.[28]

The synergetic interactions within these interfacial structures and the contribution to the photocatalytic mechanism were investigated. According to the first principle 
calculations (Figure S23), covering the hydrogen atoms on the 001 surface of $\mathrm{WO}_{3}$ can restrain the formation of extra oxygen vacancies due to coulomb repulsions, because both the absorbed hydrogen atoms and $V_{o}$ introduce extra electrons into the system. Moreover, the redistribution of $\mathrm{V}_{\mathrm{o}}$ defects actually inhibits the deep insertion of hydrogen by acting as trapping sites, which avoids the kinetic bottleneck of interfacial separation of charge carriers in thick, co-catalyst-modified nanostructures.[29] Figure 5c and 5d also show that the hydrogenated (001) surface has a different thermodynamic tendency for $\mathrm{H}_{2} \mathrm{O}$ oxidation compared to that of pristine $\mathrm{WO}_{3}$. The free energies required to dissociate $\mathrm{H}_{2} \mathrm{O}$ over a hydrogen-intercalated surface are 0.26 and $1.27 \mathrm{eV}$ lower than that required on oxygen-defective and pristine $\mathrm{WO}_{3}(001)$ surfaces. Thus, the hydrogenated $\mathrm{WO}_{3}(001)$ surface is the most energy favorable surface for oxidizing water into $\bullet \mathrm{OH}$ (Figure S24). Moreover, terminal hydroxyls $\left(\mathrm{OH}_{t}\right)$ are the dominant surface species for hydrogen-intercalated materials upon hydroxylation.[30] The facilitated adsorption of water molecules on the hydrogen-intercalated surface benefits efficient hole trapping for water oxidation.[31] 
a

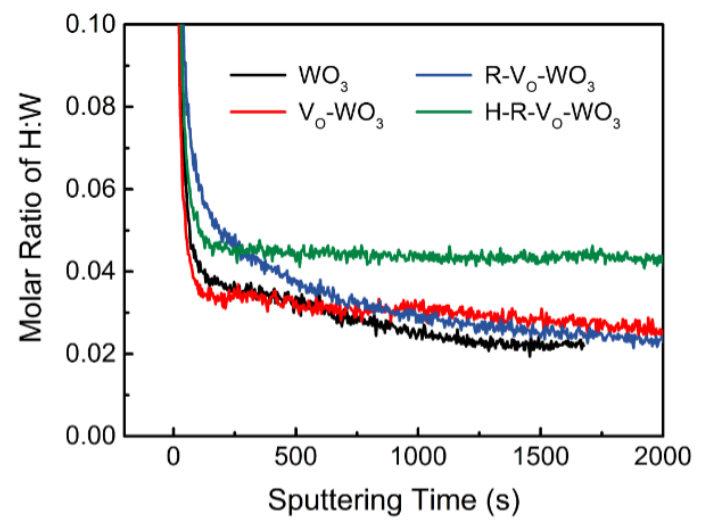

C

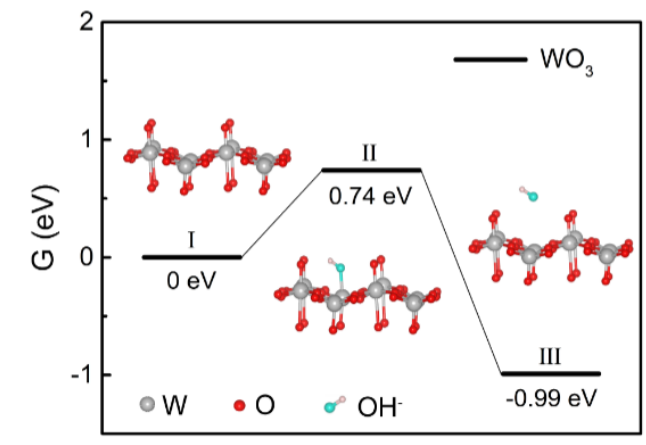

Reaction Pathway b

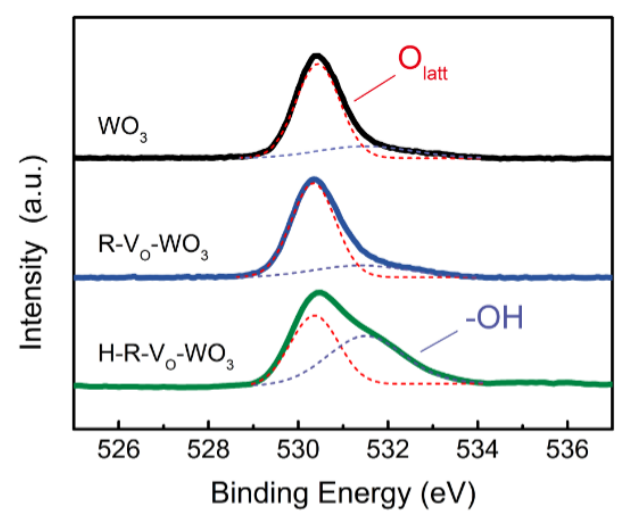

d

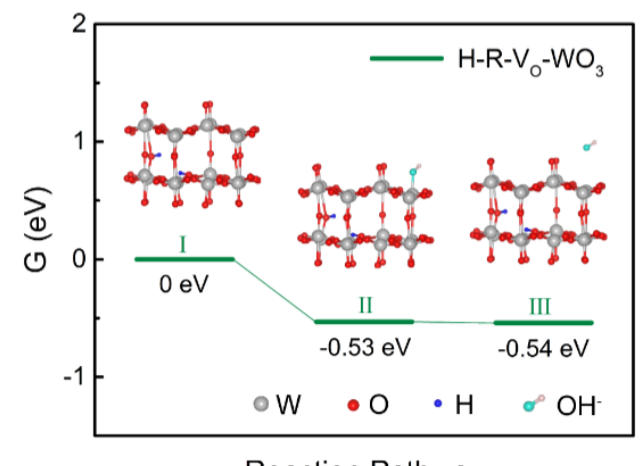

Figure 5 (a) ToF-SIMS depth profiles showing the elemental $\mathrm{H}$ content relative to $\mathrm{W}$ vs. the sputtering time; (b) O $1 \mathrm{~s}$ XPS spectra of $\mathrm{WO}_{3}, \mathrm{~V}_{\mathrm{o}}-\mathrm{WO}_{3}$ andH-R-V $-\mathrm{V}_{\mathrm{o}}-\mathrm{WO}_{3}$; (c) Schematic of water oxidationon a pristine $\mathrm{WO}_{3}$ surface; (d) Schematic of water oxidation on a $\mathrm{H}-\mathrm{R}-\mathrm{V}_{\mathrm{o}}-\mathrm{WO}_{3}$ surface.

However, $\mathrm{V}_{\mathrm{o}}$ can also result in the formation of bridging hydroxyls $\left(\mathrm{OH}_{\mathrm{b}}\right)$. [32,33] The electron-trapping nature of the hydroxyls can contribute to the interfacial activation of oxygen molecules, which is consistent with the calculated higher adsorption energy foroxygen molecules on the oxygen-defective $\mathrm{WO}_{3}(-2.63 \mathrm{eV})$ compared to that of pristine $(-1.22 \mathrm{eV})$ and hydrogenated $\mathrm{WO}_{3}(-1.86 \mathrm{eV})$ (Figure S25). From this perspective, a termination-dependent photocatalytic mechanism was conceived for the hydrogen-intercalated nonstoichiometric semiconductors and was experimentally verified using the 5,5-dimethyl-1-pyrroline-N-oxide (DMPO) spin-trapping ESR technique. Figure $6 \mathrm{a}$ and $6 \mathrm{~b}$ clearlyshow the preferential generation of $\cdot \mathrm{OH}$ (oxidation of water by holes) over the hydrogen-intercalated layer, whereas $\mathrm{O}_{2} \cdot{ }^{-}$(reduction of oxygen by electrons) was the dominant radical over 
$\mathrm{V}_{\mathrm{o}^{-}}-\mathrm{WO}_{3}$. Accordingly, the divergent performances of $\mathrm{V}_{\mathrm{o}}-\mathrm{WO}_{3}$ and $\mathrm{H}-\mathrm{WO}_{3}$ for the water oxidation reactions are shown in Figure S26. These results further confirmed the spatial separation of the charge carriers for high-efficiency, radical-specific, photo-redox reactions.

Speaking from a broader perspective, our research provides insight into the feasibility of synergistic modulation of $\mathrm{V}_{\mathrm{o}}$ migration and hydrogen intercalation for high-efficiency charge separation. The principle of controlling the spatial distribution of the nonstoichiometric layer and the charge-extracting layer is applicable to a wide variety of semiconductors. When an electrochemical reduction strategy was used for the interfacial modulation of $\mathrm{TiO}_{2}$ photoanodes, the surface amorphization and concomitant formation of $\mathrm{Ti}^{3+}$ were readily identified by the HRTEM images and EELS line scans (FigureS27). Benefitting from the surface states induced by the hydrogen intercalation (Figure S28), significantly improved photoactivities were achieved for $\mathrm{H}-\mathrm{R}-\mathrm{V}_{\mathrm{o}}-\mathrm{TiO}_{2}$ and $\mathrm{H}-\mathrm{FeOOH}-\mathrm{Fe}_{2} \mathrm{O}_{3}$ (Figure $6 \mathrm{c}$ and $6 \mathrm{~d}$ ). Therefore, this approach can be widely applied and is expected to accelerate the development of practical solar-fuel photocatalytic systems. We also hope that this method will lead to an atomistic-level understanding of the photocatalytic mechanism in more complex systems, and this research is currently underway in our laboratories. 
a

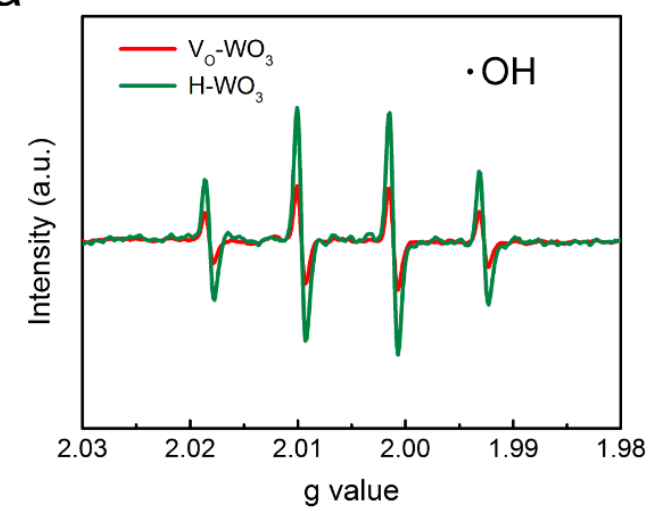

C

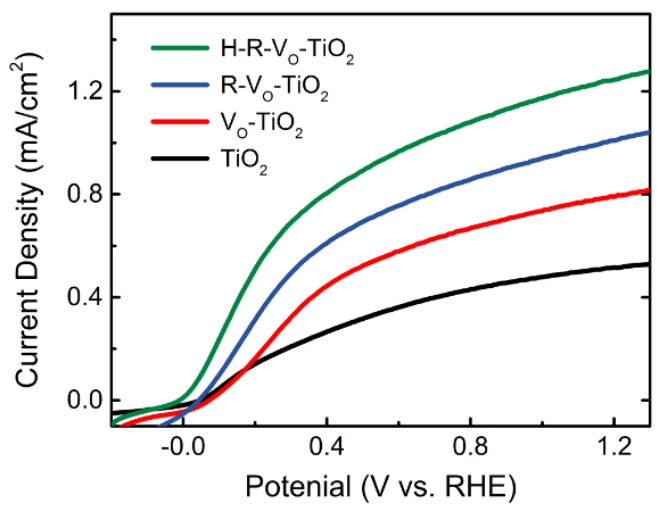

b

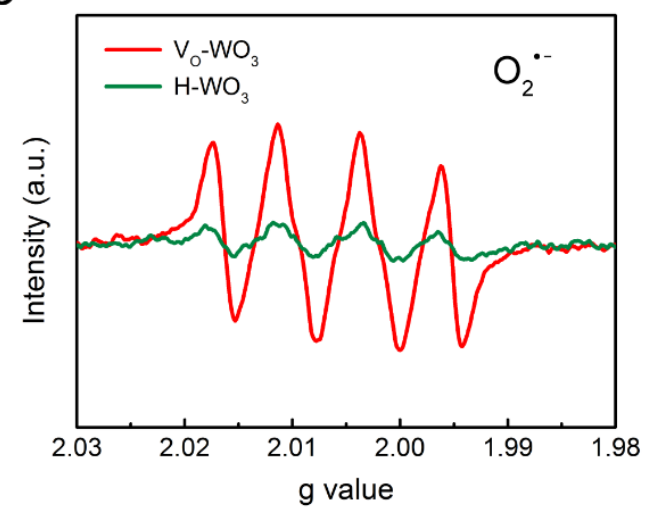

d

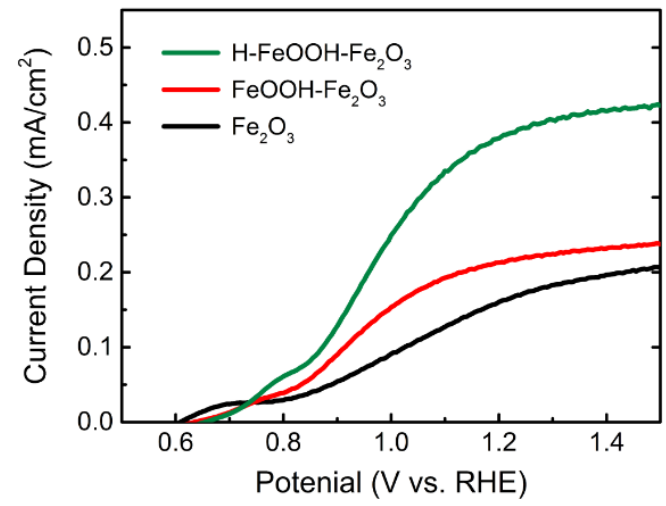

Figure 6 The DMPO-• OH(a)and DMPO- $\mathrm{O}_{2} \bullet-\left(\right.$ b) adducts produced by $\mathrm{V}_{\mathrm{o}}-\mathrm{WO}_{3}$ and $\mathrm{H}-\mathrm{WO}_{3}$; (c) Photocurrent densities of the $\mathrm{TiO}_{2}$ photoanodes with and without the surface layer modulation; (d) Photocurrent densities of the $\mathrm{Fe}_{2} \mathrm{O}_{3}$ photoanodes with and without the surface layer modulation.

\section{Conclusion}

In summary, this work presents a very interesting materialdesign principle of layer-dependent modulation to achieve high-efficiency charge separation in nonstoichiometric oxides. The synergetic combination of an oxygen-deficient layer and a hydrogen-intercalated layer was proposed, and a proof-of-concept prototype was created,manipulating photocarriers for water oxidation. The resulting photoanodes exhibited a significantly enhanced photocurrent density. Both the experimental and theoretical investigations confirmed the significant contributions of the oxygen vacancy rearrangement and the charge extraction layer to the spatial separation of the charge carriers, leading to \% charge separation and remarkably enhanced stability, thus demonstrating a great potential to overcome the charge 
separation limitation existing in robust oxide semiconductors. This novel strategy has also been demonstrated in other benchmark photocatalysts, e.g. TiO2 and Fe2O3. This new insight into the termination-dependent (oxygen-deficient and hydrogen-intercalated) interface and the corresponding photocatalytic mechanism can open a new path to develop nano-archtictures for a wide range of applications.

\section{References:}

[1] J. Liu, Y. Liu, N. Liu, Y. Han, X. Zhang, H. Huang, Y. Lifshitz, S. Lee1, J. Zhong, Z. Kang, Science 2015, 347, 970-974.

[2]Z. Zhang, J. Jr., Chem. Rev., 2012, 112, 5520-5551

[3] X. An, T. Li, J. Tang, B. Wen, Z. Hu, L. Liu, J. Qu, C. Huang, H. Liu, Adv. Energy Mater. 2016, 1502268

[4] C. Campbell, C. Peden, Science 2005, 309, 713-714.

[5] M. Tian, M. Mahjouri-Samani, G. Eres, R. Sachan, M. Yoon, M. Chisholm, K. Wang, A. Puretzky, C. Rouleau, D. Geohegan, G. Duscher, ACS Nano 2015, 9, $10482-10488$

[6] M. Setvín, U. Aschauer, P. Scheiber, Y. Li, W. Hou, M. Schmid,A. Selloni, U. Diebold, Science, 2013, 341, 988;

[7] J. Kwon, A. Sharma, J. Bain, Y. Picard, M. Skowronski, Adv. Funct. Mater. 2015, $25,2876-2883$

[8] X. Lü, A. Chen, Y. Luo, P. Lu, Y. Dai, E. Enriquez, P. Dowden, H. Xu, P. Kotula, A. Azad, D. Yarotski, R. Prasankumar, A. Taylor, J. Thompson, Q. Jia,Nano Lett., 2016, $16,5751-5755$

[9] C. Walle, J. Neugebauer, Nature 2003, 423, 626.

[10] J. Wei, H. Ji, W. Guo, A. Nevidomskyy, D. Natelson, Nature Nanaotech. 2012, 7, 357.

[11] L. Palatinus, P. Brázda,P. Boullay, O. Perez, M. Klementová, S. Petit, V. Eigner,M. Zaarour, S. Mintova, Science 2017, 355, 166-169.

[12] C. Dahlman, G. LeBlanc, A. Bergerud, C. Staller, J. Adair,D. Milliron, Nano Lett. 2016, 16, 6021-6027;

[13] W. Hong, J. Park, J. Yoon, B. Kim, J. Sohn, Y. Lee, T. Bae, S. Chang, Y. Huh, B. 
Son, E. Stach, T. Lee, M. Welland, Nano Lett. 2013, 13, 1822-1828

[14] N. Liu, V. Häublein, X. Zhou, U. Venkatesan, M. Hartmann, M. Mačković, T.

Nakajima, E. Spiecker, A. Osvet, L. Frey, P. Schmuki, Nano Lett. 2015, 15, $6815-6820$

[15] J. Morales, G. Bourret, T. Berger, K. McKenna, J. Am. Chem. Soc., 2016, 138, $15956-15964$

[16] B. Veal, S. Kim, P. Zapol, H. Iddir, P. Baldo, J. Eastman, Nature Commun. $2016,7,11892$

[17] Z. Wang, C. Yang, T. Lin, H. Yin, P. Chen, D. Wan, F. Xu, F. Huang, J. Lin, X.

Xie, M. Jiang, Adv. Funct. Mater. 2013, 23, 5444-5450

[18] G. Wang, Y. Ling, H. Wang, X. Yang, C. Wang, J. Zhang, Y. Li, Energy Environ. Sci., 2012, 5, 6180;

[19] N. Zhang, X. Li, H. Ye, S. Chen, H. Ju, D. Liu, Y. Lin, W. Ye, C. Wang, Q. Xu, J. Zhu, L. Song, J. Jiang, Y. Xiong, J. Am. Chem. Soc., 2016, 138, 8928-8935

[20] Z. Zhang, V. Quemener, C. Lin, B. Svensson, L. Brillson, Appl. Phys. Lett. 2013, $103,072107$.

[21] M. Kong, Y. Li, X. Chen, T. Tian, P. Fang., F. Zheng, X. Zhao, J. Am. Chem. Soc., 2011, 133, 16414-16417.

[22] Z. Wang, C. Yang, T. Lin, H. Yin, P. Chen, D. Wan, F. Xu, F. Huang, J. Lin, X. Xie, M. Jiang, Adv. Funct. Mater. 2013, 23, 5444-5450.

[23] C. Zhao, X. Lu, Z. Zhao, M. Li, P. Zhang, B. Wang, X. Cao, J. Zhang, G. Yuless, J. Appl. Phys. 2013, 114, 113903.

[24] J. Nowotny, M. Alim, T. Bak, M. A. Idris, M. Ionescu, K. Prince, M. Sahdan, K. Sopian, M. Teridi, W. Sigmund, Chem. Soc. Rev., 2015,44, 8424-8442.

[25] S. Altendorf, J. Jeong, D. Passarello, N. Aetukuri,M. Samant, S. Parkin, Adv. Mater. 2016, 28, 5284-5292.

[26] B. Veal1, S. Kim,w, P. Zapol, H. Iddir, P. Baldo, J. Eastman, Nature Commun. 2016, 7, 11892 .

[27] J. Wei, H. Ji, W. Guo, A. Nevidomskyy, D. Natelson, Nature Nanotech. 2012, 7, 357. 
[28] X. An, L. Zhang, B. Wen, Z. Gu, L. Liu, J. Qu, H. Liu, Nano Energy, 2017, 35, 290-298.

[29] G. Carroll, D. Zhong, D. Gamelin, Energy Environ. Sci., 2015, 8,577.

[30] D. Herlihy, M. Waegele, X. Chen, C. Pemmaraju, D. Prendergast, T. Cuk, Nature Chem. 2016, 8, 549.

[31] K. Shirai, T. Sugimoto, K. Watanabe, M. Haruta, H. Kurata, Y. Matsumoto, Nano Lett. 2016, 16, 1323-1327

[32] S. Selcuk, A. Selloni, Nature Mater. 2016, 15, 1107.

[33] H. Hussain1, G. Tocci, T.Woolcot, X. Torrelles, C. L. Pang, D. S. Humphrey, C. M. Yim, D. C. Grinter, G. Cabailh, O. Bikondoa, R. Lindsay, J. Zegenhagen, A. Michaelides, G. Thornton, Nature Mat. 2017, 16, 461.

Table of contents

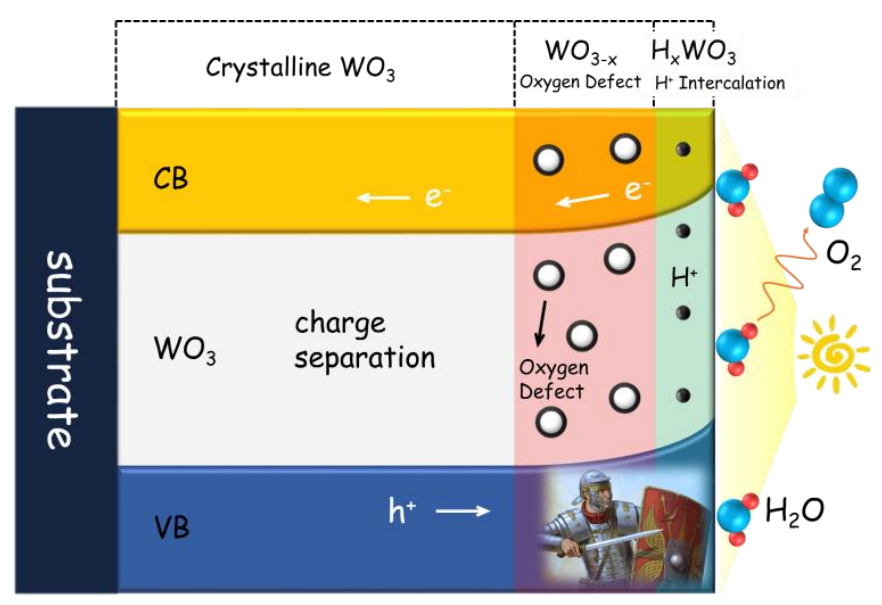

